\title{
Assessment of the Variation of the Fluid Properties and the Tar Formation by Investigating the Asphaltene Fraction
}

\author{
Honggang Zhou, Total
}

\begin{abstract}
Asphaltene rich hydrocarbon fluids often present strong variation of their composition and characteristics within a same reservoir. Sometimes, tar mat can be present. Understanding the origin and the logic of the variation of fluid properties and of the tar formation is a challenging issue because fluid properties variation as well as the tar mat formation can be due to many factors: gravity segregation, fluid loading history, biodegradation, water washing, gas striping, liquid-liquid separation, asphaltene precipitation etc. The analysis of the driving forces leading compositional and properties variation is challenging. Many potential explanations are possible and often none can be easily proven or refuted.
\end{abstract}

Recent improved understanding of the behavior of the heavy compounds such as asphaltenes may lead important progress in this area. By investigating the characteristics (and not only the quantity) of the asphaltene fraction of the oil and of the core extracts, we are now able to refute many inconsistent scenarios and bring new insights on this problem today.

In this presentation, the relevance of the data to be acquired and to be analyzed according to the objectives of the investigation will be discussed. We show that if the objective is mainly the understanding of the origin of the tar mat and it phase behavior, the information such as asphaltene content or SARA composition is poor. Investigation of the asphaltene characteristics rather than its concentration in both oil and core extract samples is recommended.

The tar mat is an asphaltene rich phase. To understand the origin of the tar mat formation, the analysis of the driving forces leading asphaltene concentration and properties variation is one of the key. Most of models proposed so far are unable of accounting consistently the intriguing solubility properties of asphaltene fractions and do not allow relevant analysis of these driving forces.

In this work, we propose an easy and powerful asphaltene characterization method for the investigation of the origin of tar mat from the point of view of phase behavior. We propose to use directly two relevant solubility properties of the asphaltene fraction instead of using complex models and chemical analyses. These relevant solubility properties are following:

Property $n^{\circ} 1$

\section{The stability of the asphaltene fraction in oil or in organic solvent depends strongly on the quality of the solvent (its composition) or of the oil (mainly its density above the bubble pressure) and is almost independent of the dilution.}

For example, in a solvent mixture containing $50 \%$ of toluene and $50 \%$ of n-heptane, if a precipitate is observed when $1 \%$ of asphaltene is added, a small precipitate will remain insoluble even if we dilute the solution with 100 times of the same toluene-heptane mixture. Inversely, if an asphaltene fraction is soluble in a solvent or in an oil at $100 \mathrm{ppm}$ level, it should be soluble even if we increase its concentration up to $1 \%$.

Consequently, it is difficult to describe the asphaltene stability in term of "solubility" which is expressed in weight fraction or in mole fraction. The term of solubility class is more appropriate. 
An easy asphaltene characterization method using the property $n^{\circ} 1$

For the description of the asphaltene solubility characteristics, we propose an easy characterization method called ASCI rating (ASCI for Asphaltene Solubility Class Index). To determine the ASCI of the asphaltene fraction in a given oil sample, we prepare 20 small flasks containing various mixtures of toluene and $n$-heptane. The flask $n^{\circ} 1$ contains $5 \%$ weight of $n$-heptane, the $\mathrm{n}^{\circ} 2$ contains $10 \%$ of heptane etc. and finally, the flask $\mathrm{n}^{\circ} 20$ contains pure n-heptane.

Few drops of the oil sample or of the solution of tar mat are then added in each of these flasks. Flasks are then shaken then stored in $50^{\circ} \mathrm{C}$ during approximately 48 hours before the observation.

If the presence of a precipitate is observed in the flask number $k$ and not observed in the flask number $k-1$, the corresponding ASCI is defined as equal to $k$.

This rating is applicable on an oil sample, on a residue of distillation as well as on a deposit sample. In the last case, the solid sample needs to be re-dissolved in a small quantity of solvent prior to the. For asphaltene rich oil, the sample can be diluted by toluene prior to the ASCI rating.

Property $n^{\circ} 2$

In spite of the insensitivity to dilution of the asphaltene stability, when the precipitation onset is reached, asphaltene precipitate progressively. This partial precipitation is not linked to the "solubility" of the whole asphaltene fraction, but to the distribution of the different asphaltenes with different solubility class inside of the same oil. In addition, a strong interaction exists between the different asphaltenes species: even at low concentration, some unstable asphaltenes can become stable if stable asphaltenes are present.

\section{As a consequence, when an asphaltene is fractionated by partial precipitation, the Asphaltene Solubility Class Index (ASCI) of the precipitated fraction should be significantly lower than the initial ASCI of the oil.}

These intriguing properties of the asphaltene can be used to analyze the phase behaviors of the asphaltenes and the tar mat in order to make difference between the asphaltenes enrichment by phase separation such as precipitation or liquid - liquid separation and the enrichment by other mechanisms such as segregation or gas striping.

In the case of asphaltene enrichment by gas striping, the ASCI of the oil should not be modified since there is no asphaltene fractionation.

In the case of gravity segregation, a continuous shift of ASCI can be observed versus depth and no difference should be observed on term of ASCI rating between the core extract and the oil sample from the same depth.

In the case of asphaltene precipitation, a strong difference should be observed between the ASCI of the tar mat or the core extract and the ASCI of the oil samples. 